\title{
Evaluation of Serum Trace Element Levels and Biochemical Parameters of COVID-19 Patients According to Disease Severity
}

\author{
Omer Faruk Kocak ${ }^{1} \cdot$ Fatma Betul Ozgeris ${ }^{2} \cdot$ Emine Parlak $^{3}$. Yucel Kadıoglu ${ }^{4}$. Neslihan Yuce ${ }^{5}$. \\ Mehmet Emrah Yaman ${ }^{4}$ Ebubekir Bakan ${ }^{5}$
}

Received: 7 July 2021 / Accepted: 30 September 2021 / Published online: 4 October 2021

(c) The Author(s), under exclusive licence to Springer Science+Business Media, LLC, part of Springer Nature 2021

\begin{abstract}
While the COVID-19 disease progresses mildly or asymptomatically in some people, its progression is severe and symptomatic in others, and it is an issue that requires a scientific response regarding the disease. The present study includes 60 people infected with COVID-19, and the cases were divided into the following groups: asymptomatic, mild, moderate, and severe. Serum Zn, Se, and Cu levels of these groups were analyzed by ICP-MS. All measurements in the patients were compared with those of 32 healthy individuals. When the patient group is compared with the control group, the serum $\mathrm{Zn}$ and $\mathrm{Se}$ concentrations were statistically low $(p<0.001)$ in the patient group. Serum Zn level decreased significantly in 4 different patient groups compared to the control group. Although the serum Se level decreased in all four patient groups compared to the control group, the change in Se level was statistically significant only in the severe and mild patient groups. This study examined serum $\mathrm{Zn}$, Se concentrations, and biochemical parameters in patients with different severity of COVID-19, compared them with healthy individuals, and revealed new targets for diagnosis and treatment by revealing those data that may be important.
\end{abstract}

Keywords COVID-19 $\cdot$ Trace elements $\cdot$ Biochemical parameters $\cdot$ ICP-MS

\section{Introduction}

It is a well-known scientific fact that viruses are some submicroscopic agents which are infectious and they multiply in living cells. They can infect all kinds of organisms such as archaea, bacteria, plants, and animals. The reason that they are named as biological agents is that, they do not have

Omer Faruk Kocak

omer.kocak@atauni.edu.tr

1 Department of Chemical Technology, Erzurum Vocational Training College, Ataturk University, Erzurum, Turkey 25240

2 Department of Nutrition and Dietetics, Faculty of Health Sciences, Atatürk University, Erzurum, Turkey 25240

3 Department of Infectious Diseases and Clinical Microbiology, Faculty of Medicine, Atatürk University, Erzurum, Turkey 25240

4 Department of Analytical Chemistry, Faculty of Pharmacy, Atatürk University, Erzurum, Turkey 25240

5 Department of Biochemistry, Faculty of Medicine, Ataturk University, Erzurum, Turkey 25240 a certain cell structure. Viruses are found in almost every ecosystem on earth and wherever there is life, and they probably have always been present in the evolutionary processes of living organisms. Depending on the type of genome they have, they are classified as DNA viruses or RNA viruses [1]. According to the International Committee on Taxonomy of Viruses, there are 7 orders, 103 families, 22 subfamilies, 455 genera, and around 2827 species in total, and more than 4000 species have not yet been classified [2]. Coronaviruses are RNA viruses belonging to the Coronaviridae family of the Nidovirales order and are very common in humans and other mammals [3]. The Coronaviridae family is genotypically and serologically divided into four genera: Alphacoronavirus, Betacoronavirus, Deltacoronavirus, and Gammacoronavirus [4]. Four types of human coronaviruses (229E, HKU1, NL63, and OC43) have been known for more than 50 years as the causes of mild to moderate respiratory tract infections [5]. However, the recently detected SARSCoV (2002) and MERS-CoV (2012) coronaviruses have caused severe acute respiratory infections and nosocomial outbreaks. Therefore, known approaches for this group of viruses have changed [3]. Then, in December 2019, a 
new coronavirus (SARS-CoV-2) was identified when the World Health Organization (WHO) China Country Office reported pneumonia of unknown etiology in Wuhan City, Hubei Province of China. The disease caused by this new virus has been named COVID-19 and has been declared a pandemic worldwide $[6,7]$. This viral infection, which has spread rapidly since the first day it was seen, continues as a global vital risk.

Although there is no definitive treatment for viruses, the function of the immune system becomes very important [8]. Trace elements act as enzyme components in biological systems or as catalysts in chemical reactions taking place in cells. Therefore, it is known that inadequate or excessive intake of a large number of elements causes a large number of diseases [9]. Trace elements are defined as metabolic and functional food components necessary to sustain life. Both trace element deficiency and excess lead to physiological disorders, and high metal concentrations can have toxic effects [10]. Trace elements play essential roles in the survival of the virus and the activation of the host's immune system [11]. For example, zinc (Zn) is antiviral against several viruses, necessary for the development of the immune system, and it inhibits the activity of the RNA-dependent RNA polymerase (RdRp) of the coronavirus [12-14]. Another prominent element for the immune system is selenium. Selenium is an essential element that plays a role in many mechanisms in the body, especially in thyroid hormone metabolism, antioxidant defense, and regulation of the immune system, and participates in many enzymes as a cofactor [15]. It has been stated that using selenium in people with low plasma selenium concentration improves cellular immunity and decreases the mutation in the viral genome [13]. While the deterioration of the immune system and increasing infection rates are associated with copper deficiency [16], the negative effects of excessive copper levels in the serum on human respiratory health are also stated [17]. There are no recommendations on copper intake against COVID-19, and it is not known whether copper-related changes are beneficial [18]. Since copper and zinc are competitively absorbed in the gastrointestinal tract, high doses of zinc may cause $\mathrm{Cu}$ deficiency in healthy individuals [19], suggesting that the $\mathrm{Zn} / \mathrm{Cu}$ ratio may be an important parameter in the follow-up of diseases. These properties of $\mathrm{Zn}, \mathrm{Se}$, and $\mathrm{Cu}$ elements show that they may be important for diagnosing and treating COVID-19 disease.

COVID-19 is the leading cause of death from viral infections in the world today. However, some people experience the disease in a mild asymptomatic phase, while others experience the disease in a severe symptomatic phase, which is another mystery of the disease that awaits scientific answers [20, 21]. In our literature review, we found studies comparing urine [22] and serum trace element levels [21, 23] and biochemical parameters (with another disease) [24] of
COVID-19 patients with control groups. However, no study has been found showing how COVID-19 disease changes simultaneously with both biochemical parameters and serum trace element concentrations, according to the severity of the disease.

Our study divided patients with confirmed SARS-CoV-2 infection into clinically asymptomatic, mild, moderate, severe, and critical groups. We aimed to describe the findings showing variation in serum trace element $(\mathrm{Zn}, \mathrm{Se}$, and $\mathrm{Cu})$ level and biochemical parameters between healthy volunteers and defined patient groups, as well as findings showing variation in serum trace element level and biochemical parameters in patient groups in correlation with the severity of the disease.

\section{Material and Methods}

\section{Study Design}

Patients and healthy people were selected from those who applied to the Infectious Diseases Department of Ataturk University Research Hospital. The groups consist of COVID-19 positive and control group with similar demographic characteristics. COVID-19 infection was diagnosed by the real-time polymerase chain reaction (RT-PCR) test through evaluation of nasopharyngeal and oropharyngeal samples taken from the patient [25]. Healthy volunteers with demographic characteristics similar to the patients, without any complaints and negative for RT-PCR, were included in the control group. All samples were taken after approval of the study by the clinical research ethics committee (No: B.30.2.ATA.0.01.00/295). All COVID-19 cases were managed with guidance by the national guideline. COVID-19 patients were divided into the following groups: asymptomatic (no symptom, no pulmonary involvement in chest imaging studies), mild (patients with symptoms such as fever, muscle and joint pain, cough, and sore throat and no respiratory distress-respiratory rate per minute $<24$, $\mathrm{SpO} 2>93 \%$ in room air-and normal chest $\mathrm{X}$-ray and/or lung tomography), moderate (patients with symptoms such as fever, muscle and joint pain, cough, and sore throat, respiratory rate $<30 / \mathrm{min}, \mathrm{SpO} 2>90$ in room air, and mild to moderate pneumonia findings on chest $\mathrm{X}$-ray or tomography), and severe (patients with symptoms such as fever, muscle and joint pain, cough, and sore throat, with tachypnea ( $\geq 30 / \mathrm{min}$ ), SpO2 level below $\leq 90 \%$ in room air, and bilateral diffuse pneumonia findings on chest X-ray or tomography) (see Table 1). 
Table 1 Demographic characteristics of the control group and patients with COVID-19

\begin{tabular}{|c|c|c|c|c|c|c|c|}
\hline & & $\begin{array}{l}\text { All patients ( } 60 \\
\text { people) }\end{array}$ & $\begin{array}{l}\text { Control ( } 32 \\
\text { people) }\end{array}$ & $\begin{array}{l}\text { Asymptomatic (4 } \\
\text { people) }\end{array}$ & Mild (15 people) & $\begin{array}{l}\text { Moderate ( } 28 \\
\text { people) }\end{array}$ & Severe (13 people) \\
\hline \multicolumn{2}{|c|}{ Age, median [IQR] } & $48.8(16-96)$ & $45.5(21-82)$ & $41.25(24-56)$ & $31.9(16-72)$ & $54.1(23-96)$ & $58(39-85)$ \\
\hline \multirow[t]{2}{*}{ Sex } & Male & 32 & 11 & 2 & 13 & 13 & 4 \\
\hline & Female & 28 & 21 & 2 & 2 & 15 & 9 \\
\hline
\end{tabular}

\section{Examination of Biochemical and Clinical Profile}

Blood samples were taken from patients admitted to our hospital. While some of the blood samples were sent to the hospital laboratory for routine analysis, another portion was sent to the Atatürk University, Faculty of Medicine, Medical Biochemistry Department research laboratory for trace element analysis. Serum $\mathrm{Zn}, \mathrm{Cu}$, and Se concentrations of each patient, along with age, gender, comorbid characteristics, and biochemical parameters (WBC, ALT, AST, BUN, INR, $\mathrm{Hb}, \mathrm{PLT}, \mathrm{LDH}, \mathrm{NE} \%$, LY\%, creatinine, glucose, troponin, ESR, ferritin), were recorded.

\section{Trace Element Analysis}

To analyze trace elements by ICP-MS, a previously described method was followed with minor modifications [26]. Collected blood specimens were centrifuged, and serum was separated. A total of $0.5 \mathrm{~mL}$ of serum samples was taken and transferred into Teflon cups. A total of $9 \mathrm{~mL}$ of $\mathrm{HNO} 3$ and $1 \mathrm{~mL}$ of $\mathrm{H} 2 \mathrm{O} 2$ solution were added to them. The mixture was digested in the microwave for $120 \mathrm{~min}$ at $150{ }^{\circ} \mathrm{C}$. The volume of digested samples was adjusted to $15 \mathrm{~mL}$ by adding ultrapure water. Inductively coupled plasma mass spectrometer (ICP-MS, Agilent 7700) was used for analysis. Standard solutions of the elements to be analyzed $(\mathrm{Cu}, \mathrm{Zn}, \mathrm{Se})$ were prepared using increasing concentrations of $2 \%$ nitric acid solution. Calibration curves were drawn, and indium, scandium, germanium, and bismuth were used as internal standards to correct for deviations in the calibration curve during analysis. The ICP-MS system was operated at $1550 \mathrm{~W}$ radio frequency power, $4.3 \mathrm{~mL} / \mathrm{min}$ He flow rate, and $15 \mathrm{~L} / \mathrm{min}$ argon (Ar) plasma gas flow rate; and method conditions were determined as $1 \mathrm{~L} / \mathrm{min}$ and 0.99 L/min Ar auxiliary and carrier gas flow rates, respectively, and analyses were performed. Measured concentration values (ppb) were recorded.

\section{Statistical Analysis}

The IBM SPSS 20.0 package program was applied for analysis in the study. The Student's $t$-test was used to compare the data of the COVID-19 patients and the control group. Pearson correlation was used for correlation analysis between data. One-way ANOVA analysis was performed to examine the change in biochemical parameters and trace element levels between the control and patient groups (asymptomatic, mild, moderate, and severe). $p<0.05$ was considered significant, and $p<0.001$ was considered very significant.

\section{Results}

A total of 92 people, 60 of whom were infected and 32 of whom were healthy, were included in this study. Routine biochemical parameters and serum trace element levels of the participants were measured. The results of the measurements were statistically analyzed using the Student's $t$-test (Table 2).

When the biochemical parameters of COVID-19 patients were compared with the control group, Ne\% $(p=0.013)$, HB $(p=0.003)$, ferritin $(p=0.005), \mathrm{LDH}(p<0.001)$, and glucose $(p<0.001)$ values increased, while LY\% and PLT values decreased. When the patient group is compared with the control group, the serum $\mathrm{Zn}$ and Se concentrations are statistically very significantly $(p<0.001)$ low in the patient group (Fig. 1).

As a result of Pearson correlation analysis to understand whether there is a correlation between the biochemical parameters of the patient group and serum trace element concentrations, it has been seen that there is a positive relationship between ESR value and $\mathrm{Cu}$ value, a significant negative relationship between Se value and LDH and Troponin, and a very significant positive relationship between $\mathrm{Zn} / \mathrm{Cu}$ ratio and $\mathrm{Hb}$ value (Table 3 ).

After the COVID-19 patients were divided into four groups according to their severity (asymptomatic, mild, moderate, and severe), the one-way ANOVA test was performed to determine a statistical difference between the groups and the control group in terms of biochemical parameters and trace elements (Table 4).

According to one-way analysis of variance; Parameters that differed significantly between the control and patient groups and within the patient groups were determined. Serum $\mathrm{Zn}$ levels changed significantly in all patient groups compared to the control group. Serum Zn level decreased significantly in 4 different patient groups compared to the control group. Although the serum Se level decreased in 
Table 2 Changes in biochemical parameters and serum trace element concentrations in the patient and control groups

\begin{tabular}{|c|c|c|c|c|c|c|}
\hline \multirow[t]{2}{*}{ Parameter } & \multicolumn{2}{|c|}{ COVID-19 (+) } & \multicolumn{2}{|l|}{ Control } & \multirow[t]{2}{*}{ Direction } & \multirow[t]{2}{*}{$p$ value } \\
\hline & Median & SD & Median & $\mathrm{SD}$ & & \\
\hline WBC $(\mu \mathrm{L})$ & 6370.33 & 2529.95 & 7147.19 & 1661.31 & $\downarrow$ & NS \\
\hline NE\% & 64.14 & 12.24 & 57.55 & 10.95 & $\uparrow$ & 0.013 \\
\hline LY\% & 24.41 & 10.22 & 31.68 & 10.84 & $\downarrow$ & 0.002 \\
\hline $\mathrm{Hb}(\mathrm{g} / \mathrm{dL})$ & 14.84 & 1.68 & 13.58 & 2.21 & $\uparrow$ & 0.003 \\
\hline $\operatorname{PLT}(\mu \mathrm{L})$ & $229,433.33$ & $65,348.19$ & $275,250.00$ & $105,778.53$ & $\downarrow$ & 0.012 \\
\hline BUN (mg/dL) & 14.97 & 5.29 & 13.74 & 3.65 & $\uparrow$ & NS \\
\hline Creatinine (mg/dL) & 0.82 & 0.23 & 0.76 & 0.16 & $\uparrow$ & NS \\
\hline AST (U/L) & 26.48 & 8.75 & 27.21 & 16.89 & $\downarrow$ & NS \\
\hline ALT (U/L) & 24.13 & 16.55 & 29.75 & 27.12 & $\downarrow$ & NS \\
\hline LDH (U/L) & 286.17 & 108.33 & 199.97 & 62.49 & $\uparrow$ & 0.000 \\
\hline Glucose (mg/dL) & 115.47 & 45.60 & 84.00 & 14.38 & $\uparrow$ & 0.000 \\
\hline PT (sec) & 14.68 & 1.93 & 14.45 & 1.67 & $\uparrow$ & NS \\
\hline PTT (sec) & 28.90 & 2.16 & 28.29 & 5.34 & $\uparrow$ & NS \\
\hline INR & 1.09 & 0.15 & 1.09 & 0.12 & $\downarrow$ & NS \\
\hline Troponin (ng/L) & 5.27 & 7.43 & 6.96 & 0.52 & $\downarrow$ & NS \\
\hline ESR (mm) & 22.19 & 18.49 & 11.55 & 11.40 & $\uparrow$ & NS \\
\hline Ferritin $(\mathrm{ng} / \mathrm{mL})$ & 182.99 & 231.82 & 40.51 & 51.10 & $\uparrow$ & 0.005 \\
\hline $\mathrm{Cu}(\mathrm{ppb})$ & 952.48 & 388.75 & 2795.99 & 9605.09 & $\downarrow$ & NS \\
\hline $\mathrm{Zn}(\mathrm{ppb})$ & 588.17 & 195.02 & 873.4 & 335.38 & $\downarrow$ & 0.000 \\
\hline $\mathrm{Se}(\mathrm{ppb})$ & 215.34 & 49.83 & 255.23 & 42.67 & $\downarrow$ & 0.000 \\
\hline $\mathrm{Zn} / \mathrm{Cu}$ & 0.68 & 0.28 & 0.86 & 0.63 & $\downarrow$ & NS \\
\hline
\end{tabular}

$p<0.05$ was considered significant, and $p<0.001$ was considered very significant.

$W B C$ white blood cell, $A L T$ alanine aminotransferase, $A S T$ aspartate aminotransferase, $B U N$ blood urea nitrogen, $I N R$ international normalized ratio, $H b$ hemoglobin, $P L T$ platelet, $L D H$ lactate dehydrogenase, $N E$ neutrophil, $L Y$ lymphocyte, $S D$ standard deviation, $N S$ not significant.
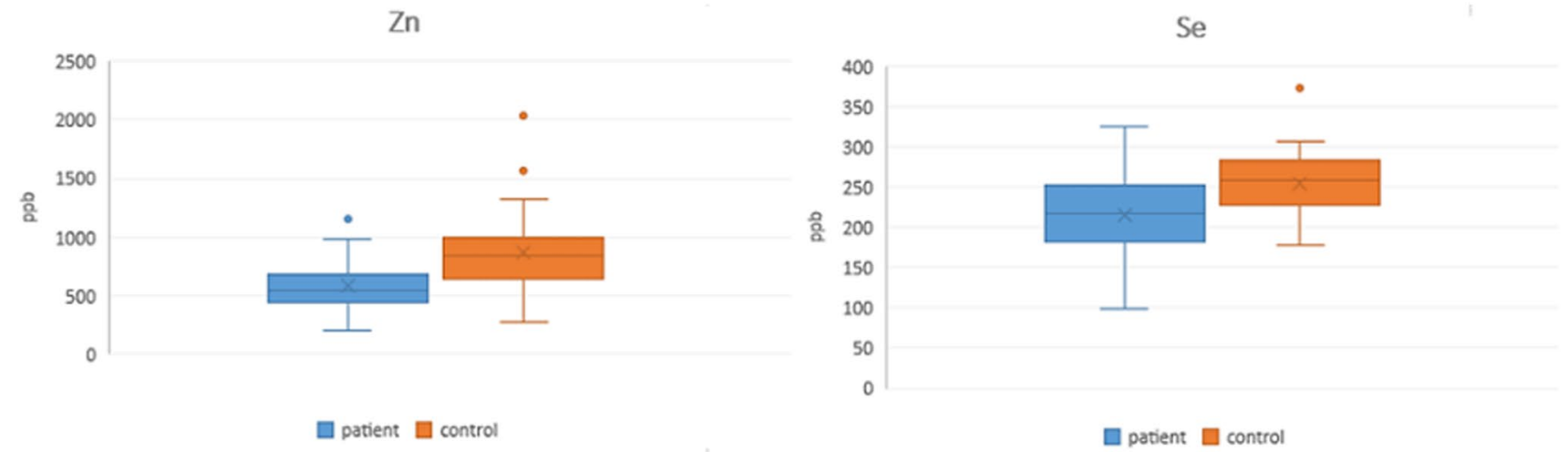

Fig. 1 The change of serum $\mathrm{Zn}$ and Se concentrations in COVID-19 patients compared to the control group $(p<0.001)$

all four patient groups compared to the control group, the change in Se level only in the severe and mild patient groups was statistically significant.

While NE\% values of severe patients decreased significantly compared to both mild and control groups, LY\% values of severe patients showed a significant decrease compared to the control group. Ferritin and LDH values of the severe patients group increased significantly compared to control group, while PLT values decreased significantly. ESR values were statistically significantly lower in Severe and Asymptomatic patients than mild, moderate, and Control groups. In addition, the mean ages of those who had the asymptomatic, mild, moderate, and severe disease were $41.25,31.93,54.11$ and 59.00 , respectively. 
Table 3 Pearson correlation analysis results of the patient group data

\begin{tabular}{|c|c|c|c|c|c|c|c|c|c|c|c|c|c|c|c|c|c|c|c|c|c|}
\hline & WBC & $\mathrm{NE} \%$ & LY\% & $\mathrm{Hb}$ & PLT & BUN & Creatinine & AST & ALT & $\mathrm{LDH}$ & Glucose & PT & PTT & INR & Troponin & ESR & Ferritin & $\mathrm{Cu}$ & $\mathrm{Zn}$ & $\mathrm{Se}$ & $\mathrm{Zn} / \mathrm{Cu}$ \\
\hline WBC & 1 & $\begin{array}{l}.288^{\text {tht }} \\
(.005) \\
\end{array}$ & $\begin{array}{l}-.142 \\
(.178) \\
\end{array}$ & $\begin{array}{l}.053 \\
(.615) \\
\end{array}$ & $\begin{array}{l}.447^{\text {tw }} \\
(.000) \\
\end{array}$ & $\begin{array}{l}.148 \\
(.167) \\
\end{array}$ & $\begin{array}{c}-.006 \\
(.959) \\
\end{array}$ & $\begin{array}{l}-.064 \\
.556) \\
\end{array}$ & $\begin{array}{l}.097 \\
. .371 \\
\end{array}$ & $\begin{array}{l}.016 \\
(.887) \\
\end{array}$ & $\begin{array}{l}.033 \\
(.760) \\
\end{array}$ & $\begin{array}{l}.154 \\
(.232) \\
\end{array}$ & $\begin{array}{l}-.164 \\
.203) \\
\end{array}$ & $\begin{array}{l}.163 \\
(.207) \\
\end{array}$ & $\begin{array}{l}-.105 \\
(.359) \\
\end{array}$ & $\begin{array}{l}.099 \\
(.453)\end{array}$ & $\begin{array}{l}.274^{*} \\
(.108) \\
\end{array}$ & $\begin{array}{l}.141 \\
.181 \\
\end{array}$ & $\begin{array}{l}.030 \\
(.776) \\
\end{array}$ & $\begin{array}{l}-.110 \\
(.298) \\
\end{array}$ & $\begin{array}{l}.142 \\
(.177) \\
\end{array}$ \\
\hline NE\% & & 1 & $\begin{array}{l}-.916^{*} \\
(.000)\end{array}$ & $\begin{array}{l}.190 \\
(.070) \\
\end{array}$ & $\begin{array}{l}.047 \\
(.658) \\
\end{array}$ & $\begin{array}{l}.134 \\
(.212) \\
\end{array}$ & $\begin{array}{l}.039 \\
(.715)\end{array}$ & $\begin{array}{l}.060 \\
(578)\end{array}$ & $\begin{array}{l}.056 \\
(606)\end{array}$ & $\begin{array}{l}.346^{2+4} \\
(.001)\end{array}$ & $\begin{array}{l}.247^{\circ} \\
(.019)\end{array}$ & $\begin{array}{l}.040 \\
(.755)\end{array}$ & $\begin{array}{l}.126 \\
.330 \\
\end{array}$ & $\begin{array}{l}-.005 \\
(.967) \\
\end{array}$ & $\begin{array}{l}.154 \\
(.179)\end{array}$ & $\begin{array}{l}.448^{+*} \\
(.000)\end{array}$ & $\begin{array}{l}.418^{+4+4} \\
(.000)\end{array}$ & $\begin{array}{l}.097 \\
. .357 \\
\end{array}$ & $\begin{array}{l}-.165 \\
(.117) \\
\end{array}$ & $\begin{array}{l}-.098 \\
(.353) \\
\end{array}$ & $\begin{array}{l}-.327^{\circ} \\
(.001) \\
\end{array}$ \\
\hline LY\% & & & 1 & $\begin{array}{l}.177 \\
(.092) \\
\end{array}$ & $\begin{array}{l}-.027 \\
(.801) \\
\end{array}$ & $\begin{array}{l}-.102 \\
(.344) \\
\end{array}$ & $\begin{array}{l}-.068 \\
(.529) \\
\end{array}$ & $\begin{array}{l}-.077 \\
(.474 \\
\end{array}$ & $\begin{array}{l}-.076 \\
(.481 \\
\end{array}$ & $\begin{array}{l}-.362^{*+1} \\
(.001) \\
\end{array}$ & $\begin{array}{l}-.227^{*} \\
(.031) \\
\end{array}$ & $\begin{array}{l}.145 \\
(.262) \\
\end{array}$ & $\begin{array}{l}-.119 \\
.358 \\
\end{array}$ & $\begin{array}{l}.098 \\
(.448) \\
\end{array}$ & $\begin{array}{l}.131 \\
(.254) \\
\end{array}$ & $\begin{array}{l}-.445^{* 0} \\
(.000) \\
\end{array}$ & $\begin{array}{l}-.393^{* m} \\
(.001) \\
\end{array}$ & $\begin{array}{l}-.099 \\
. .349 \\
\end{array}$ & $\begin{array}{l}.171 \\
(.103) \\
\end{array}$ & $\begin{array}{l}.171 \\
(.103) \\
\end{array}$ & $\begin{array}{l}.4033^{*+1} \\
(.000) \\
\end{array}$ \\
\hline $\mathrm{Hb}$ & & & & 1 & $\begin{array}{l}-.299^{*+*} \\
(.004)\end{array}$ & $\begin{array}{l}.168 \\
(.116) \\
\end{array}$ & $\begin{array}{l}.281^{\text {tot }} \\
(.008)\end{array}$ & $\begin{array}{l}.193 \\
(.072 \\
\end{array}$ & $\begin{array}{l}.200 \\
(.061\end{array}$ & $\begin{array}{l}.011 \\
(.918) \\
\end{array}$ & $\begin{array}{l}-.053 \\
(.619) \\
\end{array}$ & $\begin{array}{l}.044 \\
(.736) \\
\end{array}$ & $\begin{array}{l}.292^{*} \\
. .021\end{array}$ & $\begin{array}{l}.022 \\
(.866) \\
\end{array}$ & $\begin{array}{l}-.131 \\
(.253) \\
\end{array}$ & $\begin{array}{l}-.185 \\
(.161) \\
\end{array}$ & $\begin{array}{l}.027 \\
(.819) \\
\end{array}$ & $\begin{array}{l}-.132 \\
.210 \\
\end{array}$ & $\begin{array}{l}.015 \\
(.883)\end{array}$ & $\begin{array}{l}-.127 \\
(.227) \\
\end{array}$ & $\begin{array}{l}.142 \\
(.176) \\
\end{array}$ \\
\hline PLT & & & & & 1 & $\begin{array}{l}.114 \\
(.288) \\
\end{array}$ & $\begin{array}{r}. .157 \\
(.142) \\
\end{array}$ & $\begin{array}{l}-.154 \\
0.152 \\
\end{array}$ & $\begin{array}{l}-.079 \\
(.464 \\
\end{array}$ & $\begin{array}{l}.012 \\
(.916) \\
\end{array}$ & $\begin{array}{l}-.199 \\
(.059)\end{array}$ & $\begin{array}{l}-.080 \\
(.535)\end{array}$ & $\begin{array}{l}.217 \\
.090 \\
\end{array}$ & $\begin{array}{l}.091 \\
(.480) \\
\end{array}$ & $\begin{array}{l}-.135 \\
(.240) \\
\end{array}$ & $\begin{array}{l}-.080 \\
(.545)\end{array}$ & $\begin{array}{l}.008 \\
(.947)\end{array}$ & $\begin{array}{l}.203 \\
.052 \\
\end{array}$ & $\begin{array}{l}-.107 \\
(.310)\end{array}$ & $\begin{array}{l}.084 \\
(.423)\end{array}$ & $\begin{array}{l}-.028 \\
(.789) \\
\end{array}$ \\
\hline BUN & & & & & & 1 & $\begin{array}{l}.521^{\text {tht }} \\
(.000) \\
\end{array}$ & $\begin{array}{l}.041 \\
(.704 \\
\end{array}$ & $\begin{array}{l}-.078 \\
(.468 \\
\end{array}$ & $\begin{array}{l}.142 \\
(.195)\end{array}$ & $\begin{array}{l}.297^{2 x} \\
(.005) \\
\end{array}$ & $\begin{array}{l}.069 \\
(.592) \\
\end{array}$ & $\begin{array}{l}.086 \\
. .504 \\
\end{array}$ & $\begin{array}{l}.053 \\
(.680) \\
\end{array}$ & $\begin{array}{l}.332^{\text {th }} \\
(.003)\end{array}$ & $\begin{array}{l}.005 \\
(.973) \\
\end{array}$ & $\begin{array}{l}.363^{\text {th }} \\
(.002) \\
\end{array}$ & $\begin{array}{l}.010 \\
. .925 \\
\end{array}$ & $\begin{array}{l}.147 \\
(.170) \\
\end{array}$ & $\begin{array}{l}-.205 \\
(.053) \\
\end{array}$ & $\begin{array}{l}-.045 \\
(.676) \\
\end{array}$ \\
\hline Creatinine & & & & & & & 1 & $\begin{array}{l}.167 \\
(.120 \\
\end{array}$ & $\begin{array}{l}.047 \\
. .665 \\
\end{array}$ & $\begin{array}{l}.227^{*} \\
(.036)\end{array}$ & $\begin{array}{l}.135 \\
(.213) \\
\end{array}$ & $\begin{array}{l}.189 \\
(.141) \\
\end{array}$ & $\begin{array}{l}.021 \\
.873 \\
\end{array}$ & $\begin{array}{l}.171 \\
(.184) \\
\end{array}$ & $\begin{array}{l}.345^{*+4} \\
(.002)\end{array}$ & $\begin{array}{l}.125 \\
(.349) \\
\end{array}$ & $\begin{array}{l}.077 \\
(.522) \\
\end{array}$ & $\begin{array}{l}.072 \\
.502 \\
\end{array}$ & $\begin{array}{l}.049 \\
(.648) \\
\end{array}$ & $\begin{array}{l}-.221^{*} \\
(.038)\end{array}$ & $\begin{array}{l}.055 \\
(.606) \\
\end{array}$ \\
\hline AST & & & & & & & & 1 & $\begin{array}{l}.7899^{9+*} \\
(.000\end{array}$ & $\begin{array}{l}.357^{*+} \\
(.001) \\
\end{array}$ & $\begin{array}{l}.044 \\
(.690) \\
\end{array}$ & $\begin{array}{l}.108 \\
(.404)\end{array}$ & $\begin{array}{l}.127 \\
.325 \\
\end{array}$ & $\begin{array}{l}.093 \\
(.471) \\
\end{array}$ & $\begin{array}{l}.249^{*} \\
(.028) \\
\end{array}$ & $\begin{array}{l}.235 \\
(.075) \\
\end{array}$ & $\begin{array}{l}.067 \\
(.582) \\
\end{array}$ & $\begin{array}{l}.081 \\
. .456 \\
\end{array}$ & $\begin{array}{l}.069 \\
(.524) \\
\end{array}$ & $\begin{array}{l}.125 \\
(.244) \\
\end{array}$ & $\begin{array}{l}.024 \\
(.827) \\
\end{array}$ \\
\hline ALT & & & & & & & & & 1 & $\begin{array}{l}.005 \\
(.964)\end{array}$ & $\begin{array}{l}.014 \\
(.896) \\
\end{array}$ & $\begin{array}{l}-.014 \\
(.911) \\
\end{array}$ & $\begin{array}{l}.128 \\
.320 \\
\end{array}$ & $\begin{array}{l}.009 \\
(.946) \\
\end{array}$ & $\begin{array}{l}-.039 \\
(.734) \\
\end{array}$ & $\begin{array}{l}.037 \\
(.783) \\
\end{array}$ & $\begin{array}{l}.073 \\
(.546) \\
\end{array}$ & $\begin{array}{l}.076 \\
.482 \\
\end{array}$ & $\begin{array}{l}.135 \\
(.211) \\
\end{array}$ & $\begin{array}{l}.042 \\
(.695) \\
\end{array}$ & $\begin{array}{l}.050 \\
(.640) \\
\end{array}$ \\
\hline LDH & & & & & & & & & & 1 & $\begin{array}{l}197 \\
(.074) \\
\end{array}$ & $\begin{array}{l}.209 \\
(.111) \\
\end{array}$ & $\begin{array}{l}.002 \\
(.990 \\
\end{array}$ & $\begin{array}{l}165 \\
(.211) \\
\end{array}$ & $\begin{array}{l}.330^{+4} \\
(.004)\end{array}$ & $\begin{array}{l}.605^{+4} \\
(.000) \\
\end{array}$ & $\begin{array}{l}145 \\
(.234) \\
\end{array}$ & $\begin{array}{r}.094 \\
.393 \\
\end{array}$ & $\begin{array}{l}-.241^{*} \\
(.026) \\
\end{array}$ & $\begin{array}{l}-.331^{*+1} \\
(.002) \\
\end{array}$ & $\begin{array}{r}-.213^{\circ} \\
(.050) \\
\end{array}$ \\
\hline Glucose & & & & & & & & & & & 1 & $\begin{array}{l}146 \\
(.260) \\
\end{array}$ & $\begin{array}{l}.114 \\
. .381 \\
\end{array}$ & $\begin{array}{l}.121 \\
(.352) \\
\end{array}$ & $\begin{array}{l}.224 \\
(.050) \\
\end{array}$ & $\begin{array}{l}.058 \\
(.667) \\
\end{array}$ & $\begin{array}{l}.448^{* 1+} \\
(.000)\end{array}$ & $\begin{array}{l}.061 \\
. .568 \\
\end{array}$ & $\begin{array}{l}-.274^{\prime \prime \prime} \\
(.009) \\
\end{array}$ & $\begin{array}{l}.291^{* \prime \prime} \\
(.005)\end{array}$ & $\begin{array}{l}-.176 \\
(.098) \\
\end{array}$ \\
\hline PT & & & & & & & & & & & & 1 & $\begin{array}{l}.298^{*} \\
(.019\end{array}$ & $\begin{array}{l}.993^{* *} \\
(.000)\end{array}$ & $\begin{array}{l}-.052 \\
(.701) \\
\end{array}$ & $\begin{array}{l}-.223 \\
(.150) \\
\end{array}$ & $\begin{array}{l}-.124 \\
(.401) \\
\end{array}$ & $\begin{array}{l}-.047 \\
(.716 \\
\end{array}$ & $\begin{array}{l}.002 \\
(.985)\end{array}$ & $\begin{array}{l}-.245 \\
(.055) \\
\end{array}$ & $\begin{array}{l}.022 \\
(.867) \\
\end{array}$ \\
\hline PTT & & & & & & & & & & & & & 1 & $\begin{array}{l}.291^{*} \\
(.022) \\
\end{array}$ & $\begin{array}{l}-.174 \\
(.196) \\
\end{array}$ & $\begin{array}{l}-.218 \\
(.160) \\
\end{array}$ & $\begin{array}{l}.061 \\
(.680) \\
\end{array}$ & $\begin{array}{r}-.019 \\
(.882 \\
\end{array}$ & $\begin{array}{l}.055 \\
(.669) \\
\end{array}$ & $\begin{array}{l}-.046 \\
(.722) \\
\end{array}$ & $\begin{array}{l}.087 \\
(.503) \\
\end{array}$ \\
\hline INR & & & & & & & & & & & & & & 1 & $\begin{array}{l}-.060 \\
(.658) \\
\end{array}$ & $\begin{array}{l}-.258 \\
(.095) \\
\end{array}$ & $\begin{array}{l}.148 \\
(.317) \\
\end{array}$ & $\begin{array}{l}.034 \\
(.792 \\
\end{array}$ & $\begin{array}{l}.026 \\
(.839) \\
\end{array}$ & $\begin{array}{l}-.209 \\
(.103) \\
\end{array}$ & $\begin{array}{l}.034 \\
(.792) \\
\end{array}$ \\
\hline Troponin & & & & & & & & & & & & & & & 1 & $\begin{array}{l}.345^{* 4} \\
(.010)\end{array}$ & $\begin{array}{l}.095 \\
(.462) \\
\end{array}$ & $\begin{array}{l}.019 \\
.870 \\
\end{array}$ & $\begin{array}{l}149 \\
(.193) \\
\end{array}$ & $\begin{array}{l}.194 \\
(.089) \\
\end{array}$ & $\begin{array}{l}.078 \\
(.499) \\
\end{array}$ \\
\hline ESR & & & & & & & & & & & & & & & & 1 & $\begin{array}{l}.127 \\
(.364) \\
\end{array}$ & $\begin{array}{l}.273^{*} \\
(.037 \\
\end{array}$ & $\begin{array}{l}-.083 \\
(.530) \\
\end{array}$ & $\begin{array}{l}-.197 \\
(.134) \\
\end{array}$ & $\begin{array}{l}-.244 \\
(.062) \\
\end{array}$ \\
\hline Ferritin & & & & & & & & & & & & & & & & & 1 & $\begin{array}{l}-.072 \\
. .540 \\
\end{array}$ & $\begin{array}{l}.148 \\
(.208)\end{array}$ & $\begin{array}{l}-.304^{\text {t+t }} \\
(.008)\end{array}$ & $\begin{array}{l}.041 \\
(.729) \\
\end{array}$ \\
\hline $\mathrm{Cu}$ & & & & & & & & & & & & & & & & & & 1 & $\begin{array}{l}146 \\
(.164) \\
\end{array}$ & $\begin{array}{l}163 \\
(.121) \\
\end{array}$ & $\begin{array}{l}-.202 \\
(.053) \\
\end{array}$ \\
\hline $\mathrm{Zn}$ & & & & & & & & & & & & & & & & & & & 1 & $\begin{array}{l}.3288^{*+1} \\
(.001)\end{array}$ & $\begin{array}{l}.339^{9+*} \\
(.001)\end{array}$ \\
\hline $\mathrm{Se}$ & & & & & & & & & & & & & & & & & & & & 1 & $\begin{array}{l}-.031 \\
(.772) \\
\end{array}$ \\
\hline $\mathrm{Zn} / \mathrm{Cu}$ & & & & & & & & & & & & & & & & & & & & & 1 \\
\hline
\end{tabular}

$p$ values are shown in brackets below the coefficients. ${ }^{*} p<0.05, * * p<0.01$.

Table 4 One-way ANOVA test results

\begin{tabular}{|c|c|c|c|c|c|c|}
\hline Parameters & Control & Asymptomatic & Mild & Moderate & Severe & $p$ value \\
\hline NE $\%$ & $57.55 \pm 10.95^{\mathrm{a}}$ & $67.20 \pm 13.88^{\mathrm{a}, \mathrm{b}}$ & $58.02 \pm 9.25^{\mathrm{a}}$ & $63.91 \pm 13.06^{\mathrm{a}, \mathrm{b}}$ & $70.75 \pm 10.41^{\mathrm{b}}$ & 0.005 \\
\hline LY\% & $31.68 \pm 10.84^{\mathrm{b}}$ & $23.75 \pm 9.56^{\mathrm{a}, \mathrm{b}}$ & $29.16 \pm 8.62^{\mathrm{a}, \mathrm{b}}$ & $24.11 \pm 11.57^{\mathrm{a}, \mathrm{b}}$ & $19.77 \pm 7.12^{\mathrm{a}}$ & 0.004 \\
\hline $\operatorname{PLT}(\mu \mathrm{L})$ & $275,250.00 \pm 105,778.53^{\mathrm{b}}$ & $230,000.00 \pm 43,825.41^{\mathrm{a}, \mathrm{b}}$ & $233,200.00 \pm 53,776.26^{\mathrm{a}, \mathrm{b}}$ & $244,392.86 \pm 67,941.88^{a, b}$ & $192,692.31 \pm 68,944.89^{a}$ & 0.044 \\
\hline $\mathrm{ESR}(\mathrm{mm})$ & $11.55 \pm 11.40^{\mathrm{a}}$ & $31.67 \pm 36.47^{\mathrm{b}}$ & $10.60 \pm 13.01^{\mathrm{a}}$ & $16.55 \pm 13.02^{\mathrm{a}}$ & $38.47 \pm 14.65^{\mathrm{b}}$ & 0.000 \\
\hline Ferritin $(\mathrm{ng} / \mathrm{mL})$ & $40.51 \pm 51.10^{\mathrm{a}}$ & $97.93 \pm 54.18^{\mathrm{a}, \mathrm{b}}$ & $123.26 \pm 94.73^{\mathrm{a}, \mathrm{b}}$ & $194.01 \pm 294.84^{\mathrm{a}, \mathrm{b}}$ & $249.94 \pm 221.27^{b}$ & 0.025 \\
\hline $\mathrm{Zn}(\mathrm{ppb})$ & $873.44 \pm 335.38^{\mathrm{b}}$ & $648.74 \pm 123.52^{\mathrm{a}}$ & $600.64 \pm 181.40^{\mathrm{a}}$ & $569.07 \pm 220.54^{\mathrm{a}}$ & $564.73 \pm 180.87^{\mathrm{a}}$ & 0.000 \\
\hline $\mathrm{Se}(\mathrm{ppb})$ & $255.23 \pm 42.67^{b}$ & $236.17 \pm 52.82^{\mathrm{a}, \mathrm{b}}$ & $196.85 \pm 41.04^{\mathrm{a}}$ & $226.15 \pm 48.79^{\mathrm{a}, \mathrm{b}}$ & $206.97 \pm 57.18^{\mathrm{a}}$ & 0.000 \\
\hline
\end{tabular}

Tukey's test was used as a multiple comparison test. Values are presented as mean \pm standard deviation. Letters a-b refer to statistically different data among experimental groups.

\section{Discussion}

COVID-19 progresses with highly variable clinical findings. A group of patients is completely asymptomatic. In another group of patients, a severe disease requiring intensive care is observed due to severe hypoxemia and respiratory failure [27]. Quantitative measurements showing the pathological development process are extremely important to classify patients according to disease severity and apply effective treatment quickly. This study examined serum $\mathrm{Zn}, \mathrm{Se}$, and $\mathrm{Cu}$ concentrations and biochemical parameters in patients with different severity of COVID-19, compared them with healthy individuals, and revealed new targets for diagnosis and treatment by revealing those data that may be important. To the best of our knowledge, this is the first study in which serum trace element levels ( $\mathrm{Zn}, \mathrm{Se}, \mathrm{Cu}$ ) and biochemical parameters were evaluated depending on the severity of COVID-19 disease. In our study, trace elements and biochemical parameters, especially statistically significant, are discussed below.

$\mathrm{Zn}$ is involved in the development and maturation of immune cells and the inflammatory response. Zinc acts as a cofactor of the cellular mechanisms of replicative and transcriptional enzyme systems such as DNA and RNA polymerases, respectively [28]. In infected cells, the processing of coronavirus replicase polyproteins has been shown to be inhibited by $\mathrm{Zn}$ through inhibitory proteolytic mechanisms [29]. According to Sattar et al. [30], zinc intake with azithromycin and chloroquine accelerated the recovery rate in COVID-19 patients. Also, in another study [31], modulation of ACE-2 receptor levels with $\mathrm{Zn}$ therapy was considered a 
potential therapeutic strategy in the treatment of COVID-19. By means of this information, in our study, when the patient group (regardless of severity) and the healthy group were examined, we can explain the very significantly low serum $\mathrm{Zn}$ concentration in the patient group $(p<0.001)$. Moreover, as a result of the ANOVA test performed to examine the severity of the disease, it was observed that the serum $\mathrm{Zn}$ levels of all patient groups were significantly lower than the control group. Although the serum $\mathrm{Zn}$ levels were not statistically significant among the disease groups, it was observed that they decreased depending on the severity in the severe, moderate, mild, and asymptomatic groups, showing the correlation between disease severity and serum $\mathrm{Zn}$ change (Fig. 2).

It is known that selenium can optimize both innate and acquired immunity. Selenium deficiency has been shown to reduce the immune system and promote the pathogenicity of coronaviruses. It inhibits virus replication in host cells. The virulence of SARS-CoV is mainly due to its ability to penetrate pneumocytes [14]. One study showed that Se deficiency in Hubei Province increased the virulence of SARSCoV-2 pathogenicity [32]. Our study observed that the mean serum Se levels of the patients with positive COVID-19 test were significantly lower than the healthy ones. In addition, when the patients were grouped (ANOVA), it was observed that the healthy and asymptomatic patients had significantly higher serum Se values than the mild, moderate, and severe patients, suggesting that serum Se level is important in asymptomatic treatment of the disease.

As a result of the correlation analysis, we performed to determine the relationship between biochemical parameters and serum trace element concentrations of the patients, and it was observed that the amount of Se was significantly negatively correlated with $\mathrm{LDH}$ and troponin values. $\mathrm{LDH}$ is a cytoplasmic enzyme, and its extracellular presence indicates cell damage and death [33]. LDH is elevated in peripheral blood due to ischemia, extreme heat, cold, dehydration, chemical poisoning, and infectious toxins [34]. In the study of Tao Chen et al. [35] on COVID-19, mortality developed in 113 of 274 patients, and the other patients recovered. LDH was significantly higher in the mortal patient group. Charmin Wu et al. [36] found that LDH is significantly higher in the group that developed ARDS in patients diagnosed with COVID-19, compared to the group that did not. At the same time, LDH value was found to be higher in patients with a mortal course in the ARDS group. In another study, patients were divided into two groups with mild disease (no imaging findings and no pneumonia) and severe disease, and LDH levels were found to be significantly higher in the severe group [37]. This information explains why the LDH value is significantly higher in severe patients in the ANOVA test than the control group and why LDH has a negative correlation with Se as a result of the correlation analysis. Another parameter with which serum Se level was negatively correlated was troponin. It has been stated in COVID-19 studies that patients with high troponin levels are more likely to be admitted to intensive care and have a higher in-hospital mortality rate [38], explaining the decrease in the amount of troponin as the level of Se increases (Fig. 3).

Impairment of the immune system and increased infection rate have been associated with copper deficiency. Although excessive copper level in serum has adverse effects on human respiratory health, there is no recommendation on the amount of copper intake against COVID-19, and it is not known whether copper-related changes are beneficial [14, 18]. In our study, the serum $\mathrm{Cu}$ level did not show a statistically significant difference between the healthy and patient groups. However, the serum $\mathrm{Cu}$ levels of the patient groups are correlated with the ESR value. Although some studies [39] stated that ESR was effective in predicting pneumonia, Gong et al. [40] stated in their study that ESR was not related to the severity of the disease in patients with COVID19 pneumonia. We found that asymptomatic and severely ill patients showed similar ESR values. As a result of our
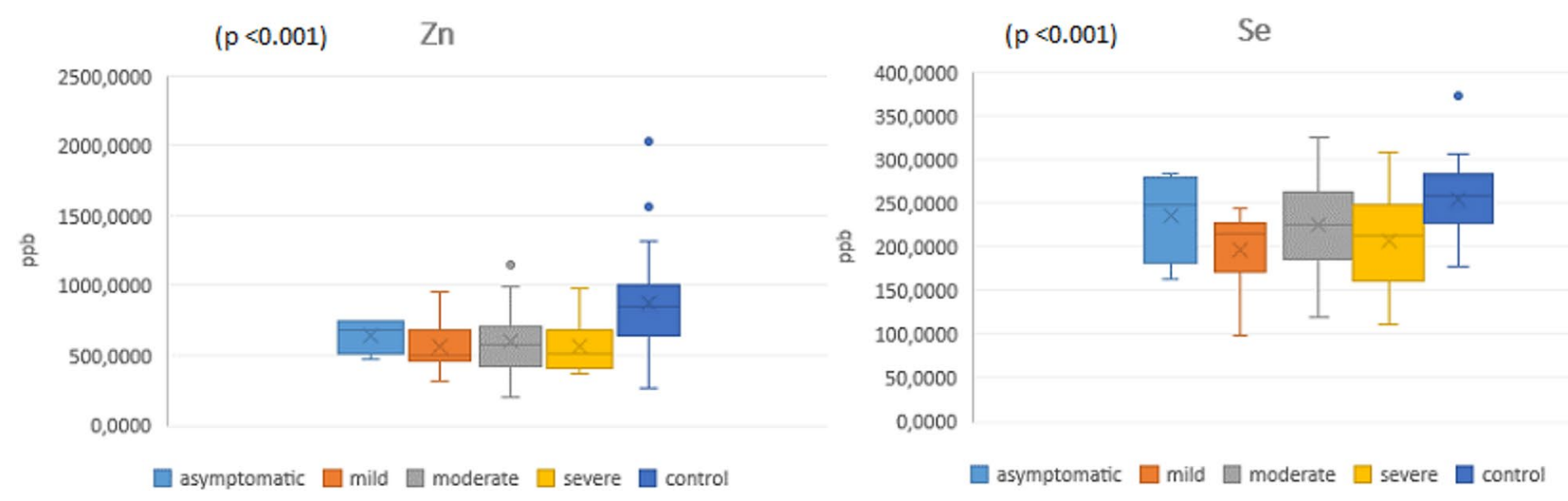

Fig. 2 The change of serum $\mathrm{Zn}$ and Se concentrations in the control group and in the subgroups of COVID-19 patients 


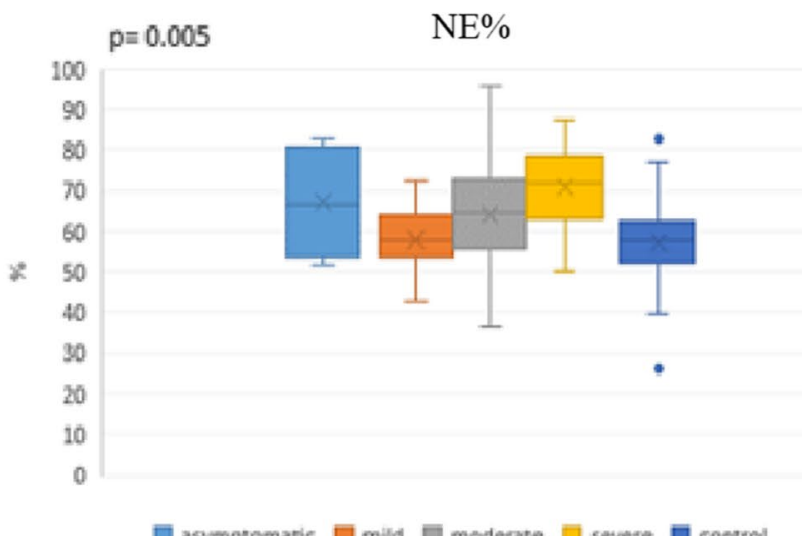

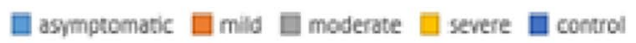
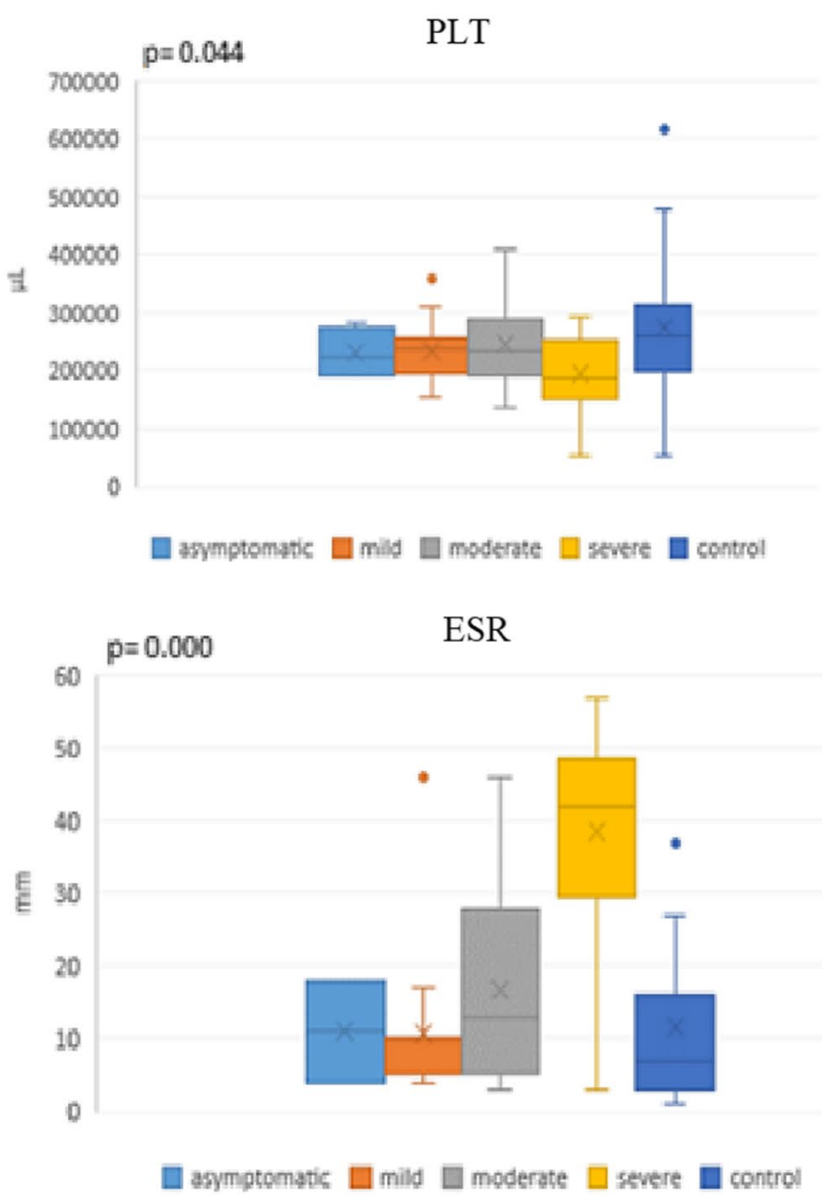
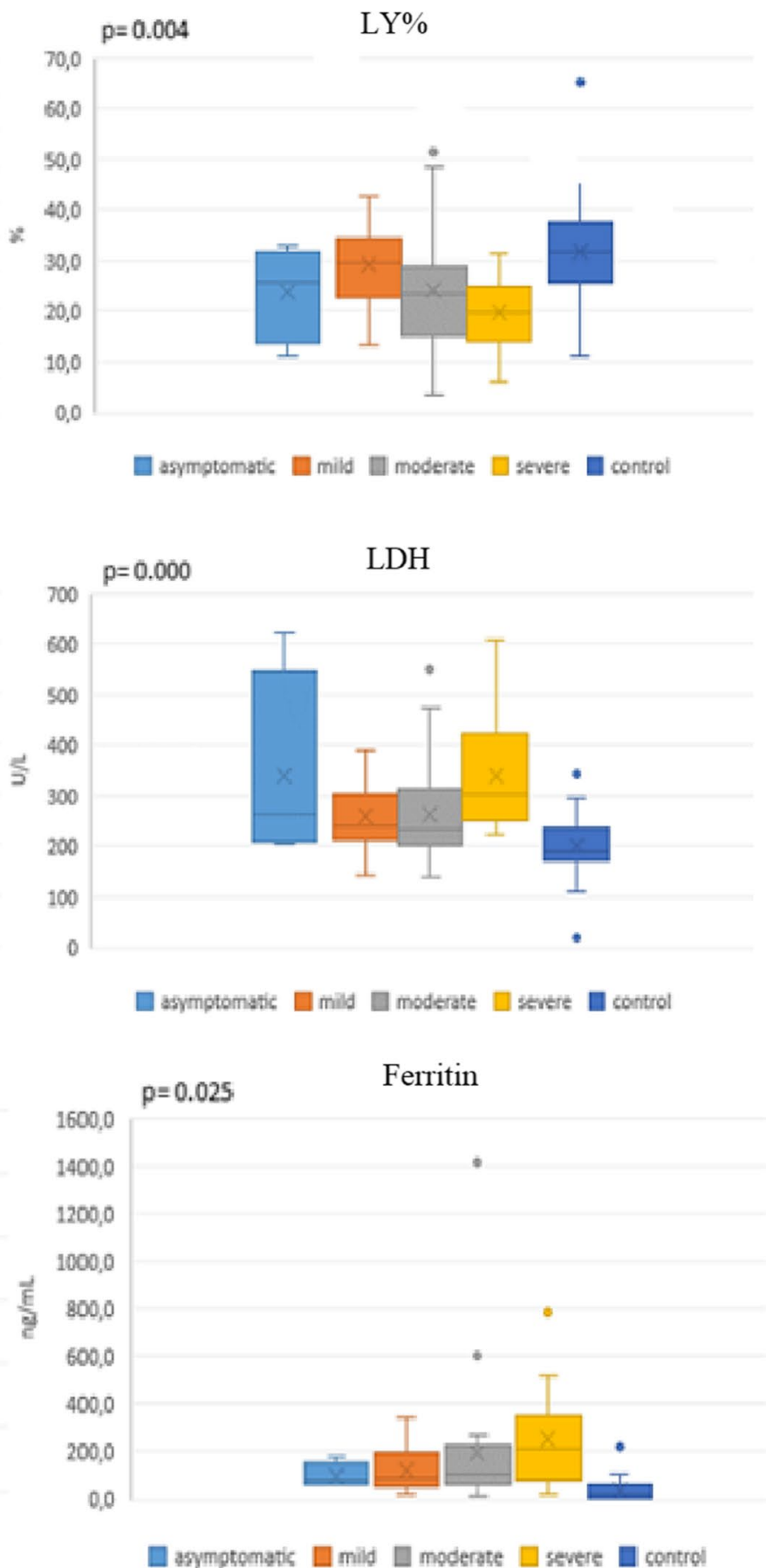

Fig. 3 The change of biochemical parameters in the control group and in the subgroups of COVID-19 patients

ANOVA test, the ESR values of asymptomatic and severe patients showed significant differences compared to the ESR values of mild, moderate, and healthy groups. These results show that the ESR value cannot be associated with disease severity as in previous studies.

In humans, copper is involved in the structure of cytochrome C-oxidase and in oxidative stress-related steps, but high copper levels may have adverse effects during inflammation. The balance between zinc and copper is important for zinc to neutralize the adverse effects that copper can cause $[24,41]$. Therefore, we thought that the $\mathrm{Zn} / \mathrm{Cu}$ ratio would be worth examining. In our study, the $\mathrm{Zn} / \mathrm{Cu}$ ratio gave a significant result neither between COVID-19 patients and the healthy group nor in terms of the severity of the disease. However, the result of the correlation analysis showed a very significant correlation with 
the $\mathrm{Hb}$ value of the $\mathrm{Zn} / \mathrm{Cu}$ ratio. In addition, $\mathrm{Hb}$ showed a significant increase in COVID-19 patients compared to the control group. Considering that COVID-19 patients have an increased risk of cardiovascular disease [6], both $\mathrm{Zn}$ deficiency and $\mathrm{Cu}$ excess (decreased $\mathrm{Zn} / \mathrm{Cu}$ ) are associated with cardiovascular disease risk [42]. Hb level is important for cardiovascular diseases [43]. The reason why the $\mathrm{Hb}$ value increases in COVID-19 patients and the $\mathrm{Hb}$ values are very significantly correlated with the $\mathrm{Zn} / \mathrm{Cu}$ ratio can be understood.

\section{Conclusion}

This study examined serum Zn, Se concentrations, and biochemical parameters in patients with different severity of COVID-19, compared them with healthy individuals, and revealed new targets for diagnosis and treatment by revealing those data that may be important. This study showed that serum $\mathrm{Zn}$ and Se values were significantly lower in COVID19 patients compared to the control group. One can conclude that serum $\mathrm{Zn}$ level could be determinant in estimating the severity of the disease, and serum Se level could be important in undergoing the disease asymptomatically. In addition, our study showed that NE\%, Hb, LDH, glucose, and ferritin values were higher in COVID-19 patients compared to the control group, while the LY\% and PLT values were lower than the control group. NE\%, LY\%, LDH, PLT, and ferritin values have been shown to be important in determining the severity of the disease. To the best of our knowledge, this is the first study in which serum trace element levels and biochemical parameters were evaluated depending on the severity of COVID-19 disease. We think that our study will be a guide for studies on the diagnosis and treatment of COVID-19 disease.

Acknowledgements The authors thank all the medical care workers who participated in collecting samples.

Data Availability Not applicable.

Code Availability Not applicable.

\section{Declarations}

Ethical Approval This study has been approved by the clinical research ethics committee of Ataturk University, Faculty of Medicine and Turkish Ministry of Health (No: B.30.2.ATA.0.01.00/295).

Consent to Participate Not applicable.

Consent for Publication Not applicable.

Conflict of Interest The authors declare no competing interests.

\section{References}

1. Şahin F and Demir S (2020) Virüsler, Viral Pandemileri Etkileyen Faktörler ve Sonuçları. Muzaffer Şeker, Ali Özer ve Cem Korkut. Türkiye Bilimler Akademisi 55-76

2. "ICTV Master Species List 2013 v2" http://talk.ictvonline.org/ files/ictv_documents/m/msl/4911.aspx. Accessed 8 Sept 2021

3. Bulut C, Kato Y (2020) Epidemiology of COVID-19. Turkish J Med Sci 50(SI-1):563-570

4. Chen $\mathrm{Y}$ et al (2020) Emerging coronaviruses: genome structure, replication, and pathogenesis. J Med Virol 92(4):418-423

5. Fung TS, Liu DX (2019) Human coronavirus: host-pathogen interaction. Annu Rev Microbiol 73:529-557

6. Huang $\mathrm{C}$ et al (2020) Clinical features of patients infected with 2019 novel coronavirus in Wuhan. China The Lancet 395(10223):497-506

7. Hanaei S and Rezaei N (2020) COVID-19: developing from an outbreak to a pandemic. Arch Med Res

8. Calder PC (2020) Nutrition, immunity and COVID-19. BMJ Nutrition, Prevention \& Health 3(1):74

9. Kocak OF, Albayrak M (2021) Analysis of trace elements in FFPE colon tissue specimens using ICP-MS. Int J Innovative Res Rev $5(1): 10-14$

10. Bánfalvi G (2011) Cellular effects of heavy metals. Springer Science \& Business Media. pp. 3-28, New York-ABD

11. de Jesus JR, de Araújo Andrade T (2020) Understanding the relationship between viral infections and trace elements from a metallomics perspective: implications for COVID-19. Metallomics 12(12):1912-1930

12. Razzaque MS (2020) COVID-19 pandemic: can maintaining optimal zinc balance enhance host resistance? Tohoku J Exp Med 251(3):175-181

13. Calder PC et al (2020) Optimal nutritional status for a well-functioning immune system is an important factor to protect against viral infections. Nutrients 12(4):1181

14. Taheri M, et al (2020) A review on the serum electrolytes and trace elements role in the pathophysiology of COVID-19. Biol Trace Element Res 1-7

15. Iglesias $P$ et al (2012) Selenium and kidney disease. J Nephrol 26(2):266-272

16. Bonham $\mathrm{M}$ et al (2002) The immune system as a physiological indicator of marginal copper status? Br J Nutr 87(5):393-403

17. Pearson $\mathrm{P}$ et al (2005) Lung function and blood levels of copper, selenium, vitamin $\mathrm{C}$ and vitamin $\mathrm{E}$ in the general population. Eur J Clin Nutr 59(9):1043-1048

18. Zabetakis I et al (2020) COVID-19: the inflammation link and the role of nutrition in potential mitigation. Nutrients 12(5):1466

19. Raha $S$ et al (2020) Is copper beneficial for COVID-19 patients?. Medical hypotheses 142:109814

20. Shi Y et al (2020) COVID-19 infection: the perspectives on immune responses. Nature Publishing Group

21. Jothimani D et al (2020) COVID-19: poor outcomes in patients with zinc deficiency. Int J Infect Dis 100:343-349

22. Zeng HL et al (2021) Urinary trace elements in association with disease severity and outcome in patients with COVID-19. Environ Res 194:110670

23. Majeed M et al (2021) An exploratory study of selenium status in healthy individuals and in patients with COVID-19 in a south Indian population: the case for adequate selenium status. Nutrition 82:111053

24. Anuk AT et al (2020) The relation between trace element status (zinc, copper, magnesium) and clinical outcomes in COVID-19 infection during pregnancy. Biol Trace Element Res 1-10

25. Tanacan A et al (2020) The rate of SARS-CoV-2 positivity in asymptomatic pregnant women admitted to hospital for delivery: 
experience of a pandemic center in Turkey. Eur J Obstet Gynecol Reprod Biol 253:31-34

26. Laur $\mathrm{N}$ et al (2020) ICP-MS trace element analysis in serum and whole blood. PloS one 15(5):e0233357

27. Ersoy EO (2020) Determination of diagnosis and disease severity, hospital and intensive care unit admission criteria in COVID-19. J Crit Intensive Care 11(Supp.):4

28. Read SA et al (2019) The role of zinc in antiviral immunity. Adv Nutr 10(4):696-710

29. Denison MR et al (1992) Intracellular processing of the N-terminal ORF 1a proteins of the coronavirus MHV-A59 requires multiple proteolytic events. Virology 189(1):274-284

30. Sattar Y et al (2020) Three cases of COVID-19 disease with colonic manifestations. Am J Gastroenterol

31. Zhang $\mathrm{H}$ et al (2020) Angiotensin-converting enzyme 2 (ACE2) as a SARS-CoV-2 receptor: molecular mechanisms and potential therapeutic target. Intensive Care Med 46(4):586-590

32. Harthill M (2011) Micronutrient selenium deficiency influences evolution of some viral infectious diseases. Biol Trace Elem Res 143(3):1325-1336

33. Drent M, Cobben N, henderson RF, Wouters EFM, van DieijenVisser M (1996) Usefulness of lactate dehydrogenase and its isoenzymes as indicators of lung damage or inflammation. Eur Resp J 9:1736-1742

34. Harbalioglu H, Omer G, and Yildirim A COVID-19 tanısı alan hastalarda yatarak tedavi görmeyi öngörmede 3 prediktör: yaş, laktat dehidrogenaz ve nötrofil/Lenfosit oranı. Pamukkale Tip Dergisi 14(1):7-7

35. Chen T, et al (2020) Clinical characteristics of 113 deceased patients with coronavirus disease 2019: retrospective study [published online March 26, 2020]. BMJ

36. Wuá CC and Caiá YX, Zhouá X, Duá C et ál (2020) Risk factors associated with acute respiratory distress syndrome and death in patients with coronavirus disease 2019 pneumonia in Wuhan, China. JAMA Intern Med 180:1-11

37. Wan $\mathrm{S}$ et al (2020) Clinical features and treatment of COVID-19 patients in northeast Chongqing. J Med Virol 92(7):797-806

38. Tersalvi G, et al (2020) Elevated troponin in patients with coronavirus disease 2019 (COVID-19): possible mechanisms. J Cardiac Failure

39. Marrie T, et al (2004) Symptoms and signs plus erythrocyte ESRentation rate or C-reactive protein predicted pneumonia in lower respiratory tract infection Contributions of symptoms, signs, erythrocyte ESRentation rate, and C-reactive protein to a diagnosis of pneumonia in acute lower respiratory tract infection. Evidence-based Med 9(2)

40. Gong J, et al (2020) Correlation analysis between disease severity and inflammation-related parameters in patients with COVID-19 pneumonia. MedRxiv

41. Kardos $\mathbf{J}$ et al (2018) Copper signalling: causes and consequences. Cell Communication and Signaling 16(1):1-22

42. Hamasaki H, Kawashima Y, Yanai H (2016) Serum $\mathrm{Zn} / \mathrm{Cu}$ ratio is associated with renal function, glycemic control, and metabolic parameters in Japanese patients with and without type 2 diabetes: a cross-sectional study. Front Endocrinol 7:147

43. Arant CB et al (2004) Hemoglobin level is an independent predictor for adverse cardiovascular outcomes in women undergoing evaluation for chest pain: results from the National Heart, Lung, and Blood Institute Women's Ischemia Syndrome Evaluation Study. J Am Coll Cardiol 43(11):2009-2014

Publisher's Note Springer Nature remains neutral with regard to jurisdictional claims in published maps and institutional affiliations. 